Ann. Biol. anim. Bioch. Biophys., 1979, 19 (6), 1701-1711.

\title{
Protéolyse pepsique, trypsique et chymotrypsique des caséines : évolution des paramètres cinétiques en fonction de traitements thermiques, mécaniques et de leurs actions conjuguées
}

\author{
par P. COTTIN, J.-L. AZANZA, A. DUCASTAING \\ Laboratoire de Biochimie de la Nutrition, \\ Université de Bordeaux I \\ Avenue des Facultés, 33405 Talence Cedex (France).
}

Summary. Peptic, tryptic and chymotryptic casein proteolysis : Changes in the kinetic parameters by thermal and mechanical treatments and their conjugate actions.

We have shown that some thermal and mechanical treatments and their conjugate actions when applied to casein solutions changed the machaelian parameters of thepeptic tryptic and chymotryptic hydrolyses of these substrates.

1) Peptic and chymotryptic hydrolyses remained constant up to $60^{\circ} \mathrm{C}$, but increased to a maximum when the temperature was raised to $80^{\circ} \mathrm{C}$. Tryptic hydrolysis however was maximum at $60^{\circ} \mathrm{C}$. In all cases, thermal treatment at high temperatures $\left(120^{\circ} \mathrm{C}\right.$ for $20 \mathrm{~min}$ ) was followed by a decrease of $V \mathrm{max}$ and an increase of $\mathrm{Km}_{\mathrm{app}}$.

2) Mechanical treatment increased the enzymatic hydrolysis process by facilitating peptic, chymotryptic and tryptic accessibility at the proteolytic sites.

3) Mechanical treatment before a thermal one increased enzymatic hydrolysis, whatever temperature was reached. The increase of substrate affinity, as well as the increment of the hydrolysis rate might be due to physico-chemical changes, including differences in protein solubility and structure.

4) Thermal denaturation, prior fo mechanical treatment, did not alter the machaelian para meters, which remained constant and maximum. In those experimental conditions, themechanical treatment suppressed the effects normally induced by the thermal treatment.

\section{Introduction.}

Mis à part le lait cru vendu avec la mention « à faire bouillir », la commercialisation du lait implique la mise en œuvre de traitements thermiques variés visant à assainir le produit et à améliorer ses qualités de conservation (Causeret ef Mocquot. 1966).

Ces traitements plus ou moins drastiques entraînent presque toujours des effets importants sur les composés protéiques du lait et notamment sur la fraction caséinique. Ainsi lors de l'étude de la thermodénaturation des caséines $\alpha_{B}$ ef $\beta$ du lait de vache, Lorient ef Alais (1970) font état de la libération d'azote et de phosphore TCA soluble 
laissant présumer la rupture de liaisons peptidiques. Ces mêmes auteurs montrent en 1974 qu'un traifement de $120^{\circ} \mathrm{C}$ pendant $25 \mathrm{mn}$ entraîne des remaniements structuraux se traduisant par une augmentation de l'absorption dans l'ultraviolet ef une opacification des solutions. Plus récemment, et pour des températures analogues, Lorient et al. (1976) signalent une dégradation thermique des caséines, caractérisée par la libération de peptides phosphorés ef le blocage des groupes $\varepsilon-\mathrm{NH}_{2}$ de la lysine.

Camus et Sautier (1972), Osner (1975), Osner ef Johnson (1975), Lorient et Alais, (1976) ont montré que les traitements thermiques, par le biais des modifications physicochimiques des molécules de caséine, influaient sur la digestibilité de la protéine. L'appréciation de la digestibilité des protéines in vivo se faił d'ordinaire par l'évaluation de leur coefficient d'utilisation digestive : pour qu'il soit très élevé, il est nécessaire que dans un délai compatible avec la vitesse de cheminement du bol alimentaire les enzymes du tube digestif (pepsine gastrique, chymotrypsine et trypsine pancréatiques, pour ne citer que les plus importantes) s'avèrent capables de faire subir à la protéine en cause une hydrolyse totale ou sub-totale. La protéolyse de ce fait peut être considérée comme un facteur caractéristique du « travail digestif».

Le présent travail concerne l'étude des modifications in vitro des protéolyses pepsiques, trypsiques et chymotrypsiques enregistrées après que divers traitements thermiques aient été appliqués aux caséines. Compte tenu des résultats obtenus, il a paru intéressant de rechercher si d'autres procédés physiques étaient capables d'influer sur l'hydrolyse enzymatique de la solution caséinique et par là même, entraîner une augmentation de la digestibilité des solutions ainsi traitées. A cette fin, des solutions caséiniques ont été soumises à un traitement mécanique (homogénéisation à grande vitesse) en alfernance avec un traitement thermique variable en fonction du temps et de la température.

\section{Matériel et méthodes.}

La caséine a été préparée selon la méthode décrite par Robert et Warner (1944) : succession de cycles de précipitation isoélectrique $(\mathrm{pH}=4,60)$ et de redissolution.

Le degré de contamination de la fraction obtenue a été évalué par électrophorèse sur gel de polyacrylamide d'après la méthode d'Orstein et Davis (1964) (fig. 1).

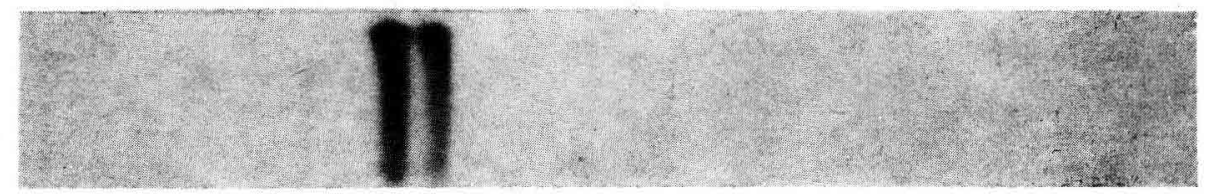

FIG. 1. - Electrophorèse en gel de polyacrylamide (7,5 p. 100) d'une prise d'essai de $30 \mu \mathrm{g}$ de la fraction caséinique préparée.

- Temps de migration : $1 \mathrm{~h} 30$ sous 200 volts et $35 \mathrm{~mA}$ en tampon TRIS-borate $\mathrm{pH}=8,60$. - Colorant : bleu de Coomassie 3 p. 100. - acide acétique 5 p. 100 - éthanol 40 p. 100 eau. - Décolorant : acide acétique 5 p. 100 - éthanol 40 p. 100 - eau.

Toute l'expérimentation a été réalisée à partir d'une solution protéique à 3 p. 100 dans l'eau distillée $(\mathrm{pH}=6,8)$. Le traitement mécanique est effectué dans un homo- 
généiseur « Virtis 60 », ultra rapide à vitesse contrôlable (de 0 à 60000 tours $/ \mathrm{mn}$ ) et a lthermodénaturation en ampoules scellées à des températures comprises entre $60^{\circ} \mathrm{C}$ et $120^{\circ} \mathrm{C}$.

Les hydrolyses par la pepsine $(100 \mathrm{mU} / \mathrm{mg})$, la chymotrypsine $20 \mathrm{U} / \mathrm{mg}$ ) et la trypsine $(3,5 \mathrm{U} / \mathrm{mg})$, enzymes commercialisées par la Firme Fluka, sont conduites à $37^{\circ} \mathrm{C}$.

A $2 \mathrm{ml}$ de solution caséinique de concentration comprise entre 3 et $30 \mathrm{~g} / \mathrm{l}$, sont ajoutés $2 \mathrm{ml}$ de solution enzymatique, contenant soit $12 \mathrm{mg}$ de pepsine dans $100 \mathrm{ml}$ d' $\mathrm{HCl} 0,15 \mathrm{~N}, \mathrm{pH}=1,90$, soit $15 \mathrm{mg}$ de trypsine dans $100 \mathrm{ml}$ de tampon. Tris- $\mathrm{HCl} 5.10^{-2} \mathrm{M}, \mathrm{pH}=8,0$ soit $15 \mathrm{mg}$ de chymotrypsine dans $100 \mathrm{ml}$ de tampon phosphate $2.10^{-2} \mathrm{M} \mathrm{PH}=7,60$. La protéolyse est stoppée au bout de $20 \mathrm{mn}$ par addition de $4 \mathrm{ml}$ de TCA à 20 p. 100.

\section{Expression des résultats.}

Les activités protéolytiques des enzymes ont été évaluées par dosage de l'azote sur les fractions protéiques TCA solubles après élimination du précipité par centrifugation à $5000 \mathrm{~g}$ pendant $15 \mathrm{mn}$. Les résultats obtenus par la méthode de Berthelot automatisée au «Technicon » sont exprimés par comparaison à une courbe étalon de sulfate d'ammonium puis transformés en grammes de protéine en utilisant un facteur de conversion, matière azotée-protéine égal à 6,39.

Chaque test de digestibilité est traduit par les deux paramètres michaeliens $V_{\max }$ ef $\mathrm{Km}_{\text {app }}$ calculés d'après la représentation linéaire de Lineweaver et Burk (1934). Il faut cependant souligner que les valeurs obtenues pour $\mathrm{Km}_{\mathrm{app}}$ et $\mathrm{V}_{\max }$ ne doivent pas être considérées comme des constantes cinétiques au sens strict. En effet, dans le cas des enzymes protéolytiques, on assiste à une hydrolyse préférentielle. d'un ou plusieurs types de liaisons peptidiques. Ainsi les mesures de vitesse d'hydrolyse de la caséine correspondent à des valeurs statistiques portant sur l'hydrolyse simultanée de plusieurs liaisons peptidiques.

Les résultats obtenus demeurent cependant comparables pour les systèmes enzymatiques testés avec le même substrat.

Les conditions expérimentales des cinétiques enzymatiques à savoir, concentrations en protéine-substrat comprises entre 3 et $30 \mathrm{~g} / \mathrm{l}$, quantités d'enzyme utilisées et temps d'incubation $(20 \mathrm{mn}$ ) sont celles qui correspondent à la phase stationnaire des réactions d'hydrolyse. Pendant cette phase l'apparition des produits d'hydrolyse en fonction du temps $\left(\frac{d P}{d t}\right.$ apparent $)$ reste constante ce qui prouve que la vitesse mesurée correspond à la vitesse initiale.

Les résultats exprimés dans les tableaux, corrigés de la valeur des témoins réalisés dans des conditions identiques correspondent à la valeur moyenne de 5 mesures: ils sont donnés sous la forme $\vec{x} \pm$ ES.

\section{Modifications de la protéolyse pepsique}

1. Influence du traitement thermique. - Les paramètres cinétiques $\mathrm{Km}_{\mathrm{app}}$ et $\mathrm{Vmax}$ regroupés dans le tableau 1 montrent que dans nos conditions expérimentales (temps 
de chauffage $20 \mathrm{mn}$ ) le thermotraitement influence de façon variable la réaction d'hydrolyse. Ainsi, la vitesse maximum constante jusqu'à $60^{\circ} \mathrm{C}$ passe par une valeur optimum pour des températures voisines de $80^{\circ} \mathrm{C}$. Lorsque le thermotraitement devient plus drastique $\left(120^{\circ} \mathrm{C}\right)$ la vitesse maximum d'hydrolyse subit une diminution notable. Le $\mathrm{Km}_{\mathrm{app}}$ suit une variation inverse.

\section{TABLEAU 1}

Paramètres cinétiques de l'hydrolyse pepsique des solutions de caséine soumises à divers traitements thermiques pendant $20 \mathrm{~min}$

\begin{tabular}{ccc}
\hline $\begin{array}{c}\text { Température de } \\
\text { chauffage }\left({ }^{\circ} \mathrm{C}\right)\end{array}$ & $\mathrm{Km}_{\mathrm{app}}(\mathrm{g} / \mathrm{l})$ & $\begin{array}{c}\text { Vitesse maximum d'hydrolyse } \\
\text { (g de protéine hydrolysée/min) }\end{array}$ \\
\hline 37 & $9,09 \pm 0,30$ & $0,140 \pm 0,004$ \\
\hline 60 & $7,69 \pm 0,21$ & $0,140 \pm 0,004$ \\
\hline 80 & $6,25 \pm 0,21$ & $0,162 \pm 0,004$ \\
\hline 100 & $6,66 \pm 0,21$ & $0,153 \pm 0,004$ \\
\hline 120 & $33,30 \pm 0,50$ & $0,100 \pm 0,003$ \\
\hline
\end{tabular}

2. Influence du traitement mécanique. - Les paramètres cinétiques $K m_{a p p}$ ef $V_{\max }$ calculés à partir de la représentation de Lineweaver-Burk (fig. 2) montrent qu'un traitement mécanique influence favorablement l'activité protéolytique (tabl. 2).

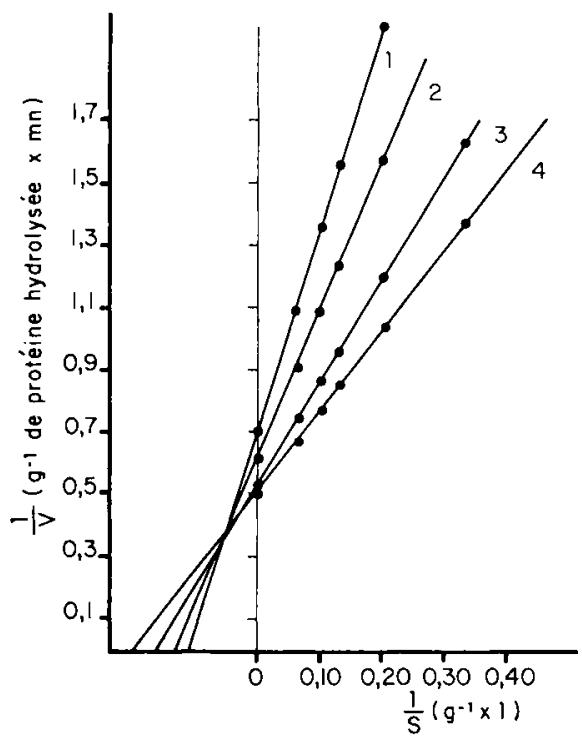

FIG. 2. - Détermination de $\mathrm{Km}_{\mathrm{app}}$ et $\mathrm{Vmax}$ lors de l'hydrolyse pepsique de la caséine native (1), mécaniquement traitée pendant $3 \mathrm{~min}$. dे 10000 et 20000 tours $/ \mathrm{min}$. (2), $20 \mathrm{~min}$. d $40000 \mathrm{tours} / \mathrm{min}$. (3), $20 \mathrm{~min}$. à 50000 tours $/ \mathrm{min}$. (4). 
Pour des temps d'homogénéisation relativement courts, la valeur des paramètres cinétiques évolue avec le nombre de tours par minute et reste constante pour 40000 tours $/ \mathrm{mn}$. Au contraire, si l'on prolonge la durée du traitement, $\mathrm{Km}_{\mathrm{mpp}}$ et $\mathrm{V}_{\max }$ atteignent d'emblée leurs valeurs extrêmes quelle que soit la vitesse de rotation.

TABLEAU 2

Evolution des paramètres cinétiques de l'hydrolyse pepsique des solutions de caséine mécaniquement traitées pendant 3 ef $20 \mathrm{~min}$

\begin{tabular}{|c|c|c|c|c|}
\hline \multirow{2}{*}{$\begin{array}{c}\text { Nombre } \\
\text { de tours/min. }\end{array}$} & \multicolumn{2}{|c|}{$K m_{a p p}(g / l)$} & \multicolumn{2}{|c|}{$\begin{array}{l}\text { Vitesse maximum d'hydrolyse } \\
\text { (g de protéine hydrolysée/min.) }\end{array}$} \\
\hline & $3 \mathrm{~min}$. & $20 \mathrm{~min}$. & $3 \mathrm{~min}$. & $20 \mathrm{~min}$. \\
\hline 0 & \multicolumn{2}{|c|}{$9,09 \pm 0,30$} & \multicolumn{2}{|c|}{$0,140 \pm 0,004$} \\
\hline 10000 & $7,69 \pm 0,21$ & $5,00 \pm 0,20$ & $0,160 \pm 0,004$ & $0,200 \pm 0,005$ \\
\hline 20000 & $7,69 \pm 0,21$ & $5,00 \pm 0,20$ & $0,160 \pm 0,004$ & $0,190 \pm 0,005$ \\
\hline 40000 & $6,25 \pm 0,21$ & $5,00 \pm 0,20$ & $0,190 \pm 0,005$ & $0,195 \pm 0,005$ \\
\hline 50000 & $5,00 \pm 0,20$ & $5,00 \pm 0,20$ & $0,195 \pm 0,005$ & $0,195 \pm 0,005$ \\
\hline
\end{tabular}

Dans le meilleur des cas, par rapport aux valeurs obtenues pour la solution caséinique native, le $\mathrm{Km}_{\text {app }}$ diminue de 45 p. 100 tandis que la vitesse maximum d'hydrolyse augmente de 39,2 p. 100.

3. Influence des traitements mécaniques antérieurs aux traitements thermiques. - Les effets d'une dénaturation thermique postérieure au traitement mécanique ont été évalués pour des températures comprises entre $37^{\circ} \mathrm{C}$ ef $120^{\circ} \mathrm{C}$ en suivant l'évolution des paramètres cinétiques d'hydrolyse. Les résultats regroupés dans le tableau 3 ainsi

TABLEAU 3

Paramètres cinétiques de l'hydrolyse pepsique des solutions de caséine dénaturées par traitement mécanique (3 min. à 50000 tours/min.) puis soumises à divers traitements thermiques pendant $20 \mathrm{~min}$.

\begin{tabular}{ccc}
\hline $\begin{array}{c}\text { Température de } \\
\text { chauffage }\left({ }^{\circ} \mathrm{C}\right)\end{array}$ & $\mathrm{Km}_{\text {app }}(\mathrm{g} / \mathrm{l})$ & $\begin{array}{c}\text { Vitesse maximum d'hydrolyse } \\
\text { (g de protéine hydrolysée/min.) }\end{array}$ \\
\hline 37 & $5,00 \pm 0,20$ & $0,200 \pm 0,005$ \\
\hline 60 & $5,00 \pm 0,20$ & $0,190 \pm 0,005$ \\
\hline 80 & $5,00 \pm 0,20$ & $0,195 \pm 0,005$ \\
\hline 100 & $5,00 \pm 0,20$ & $0,195 \pm 0,005$ \\
\hline 120 & $25,00 \pm 0,40$ & $0,160 \pm 0,004$ \\
\hline
\end{tabular}


que ceux du tableau 1 montrent que les solutions caséiniques ayant subi un traitement mécanique préalable réagissent différemment :

- entre $60^{\circ} \mathrm{C}$ et $100^{\circ} \mathrm{C}$, l'invariabilité des paramètres cinétiques montre qu'après un traitement mécanique, la digestibilité des caséines n'est plus dépendante de la température ;

- pour des températures plus élevées $\left(120^{\circ} \mathrm{C}\right)$ le $\mathrm{Km}$ app augmente.

En règle générale, les variations du $K m_{a p p}$ et de la vitesse d'hydrolyse causées par un traitement mécanique sont telles qu'une thermodénaturation postérieure apparaît sans effet jusqu'à $100^{\circ} \mathrm{C}$ (tabl. 2 et 3). Tout se passe comme si l'augmentation de la protéolyse pepsique était dans ce cas sous la seule dépendance de la tribodénaturation. Lorsque le traitement thermique est conduit à des températures voisines de $120^{\circ} \mathrm{C}$, le traitement mécanique antérieur n'est plus suffisant pour maintenir les paramètres de la réaction d'hydrolyse à leurs valeurs initiales.

\section{Modifications de l'activité protéolytique de la chymotrypsine et de la trypsine}

1. Influence du thermotraitement. - Les résultats expérimentaux regroupés dans le tableau 4 montrent que :

- Les paramètres de l'hydrolyse chymotrypsique présentent une évolution semblable à celle déjà obłenue dans le cas de la pepsine : $80^{\circ} \mathrm{C}$ représente encore la température optimale d'activité. A $120^{\circ} \mathrm{C}$, au contraire, l'activité hydrolytique ne représente plus que 70 p. 100 de l'activité initiale.

- Pour la trypsine, la température optimale est atteinte dès $60^{\circ} \mathrm{C}$. max reste ensuite constante jusqu'à $80^{\circ} \mathrm{C}$. A $120^{\circ} \mathrm{C}$, on retrouve une « inhibition » sensiblement identique à celle de la chymotrypsine.

- Pour ces mêmes températures $\mathrm{Km}_{a p p}$ suit une variation inverse de celle de $\mathrm{V}_{\max }$.

TABLEAU 4

Evolution des paramètres cinétiques d'hydrolyse chymotrypsique ef trypsique des solutions de caséine thermodénaturées pendant $20 \mathrm{~min}$

\begin{tabular}{|c|c|c|c|c|}
\hline \multirow[b]{2}{*}{$\begin{array}{l}\text { Température } \\
\text { chauffage }\left({ }^{\circ} \mathrm{C}\right)\end{array}$} & \multicolumn{2}{|c|}{ Hydrolyse chymotrypsique } & \multicolumn{2}{|c|}{ Hydrolyse trypsique } \\
\hline & $\mathrm{Km}_{\text {arp }}(\mathrm{g} / \mathrm{l})$ & $\begin{array}{c}\text { Vitesse maximum } \\
\text { d'hydrolyse } \\
\text { (g de protéine } \\
\text { hydrolysée/min) }\end{array}$ & $K m_{\text {app }}(g / l)$ & $\begin{array}{c}\text { Vitesse maximum } \\
\text { d'hydrolyse } \\
\text { (g de protéine } \\
\text { hydrolysée/min) }\end{array}$ \\
\hline 37 & $10.00 \pm 0,30$ & $0,240 \pm 0,007$ & $6,05 \pm 0,20$ & $0,110 \pm 0,003$ \\
\hline 60 & $10,00 \pm 0,30$ & $0,240 \pm 0,007$ & $5,45 \pm 0,20$ & $0,140 \pm 0,004$ \\
\hline 80 & $9,09 \pm 0,30$ & $0,280 \pm 0,007$ & $5,45 \pm 0,20$ & $0,140 \pm 0,004$ \\
\hline 120 & $14,28 \pm 0,50$ & $0,168 \pm 0,004$ & $7,50 \pm 0,20$ & $0,100 \pm 0,003$ \\
\hline
\end{tabular}


2. Influence du traitement mécanique. - Comme précédemment, les effets du traitement mécanique seul ont été recherchés en suivant l'évolution des paramètres d'hydrolyse. L'analyse du tableau 5 montre que la dénaturation mécanique du substrat augmente l'activité protéolytique de la chymotrypsine et de la trypsine.

\section{TABLEAU 5}

Paramètres cinétiques d'hydrolyse par la chymotrypsine ef la trypsine des solutions de caséine native ef traitées mécaniquement pendant $3 \mathrm{~min}$. à $50000 \mathrm{tr} / \mathrm{min}$

\begin{tabular}{|c|c|c|c|}
\hline & Paramètres cinétiques & Caséine native & $\begin{array}{l}\text { Caséine traitée } \\
\text { mécaniquement }\end{array}$ \\
\hline \multirow[b]{2}{*}{ Chymotrypsine } & $\mathrm{Km}_{\mathrm{app}}(\mathrm{g} / \mathrm{l})$ & $10 \pm 0,30$ & $6,66 \pm 0,21$ \\
\hline & $\begin{array}{l}\text { Vitesse maximum d'hydrolyse } \\
\text { (g de protéine hydrolysée/min) }\end{array}$ & $0,240 \pm 0,007$ & $0,380 \pm 0,008$ \\
\hline \multirow[b]{2}{*}{ Trypsine } & $\mathrm{Km}_{\mathrm{app}}(\mathrm{g} / \mathrm{l})$ & $6,05 \pm 0,20$ & $4,61 \pm 0,20$ \\
\hline & $\begin{array}{l}\text { Vitesse maximum d'hydrolyse } \\
\text { (g de protéine hydrolysée/min) }\end{array}$ & $0,110 \pm 0,003$ & $0,160 \pm 0,004$ \\
\hline
\end{tabular}

En effet, par rapport aux solutions caséiniques natives, les $\mathrm{Km}_{\mathrm{app}}$ diminuent respectivement de 33,4 et 23,8 p. 100 tandis que les vitesses d'hydrolyse augmentent de 58,3 et 45,4 p. 100 .

3. Influence d'un traifement mécanique antérieur aux traitements thermiques. - Les résultats obtenus et rassemblés dans le tableau 6 sont comparables à ceux déjà mentionnés dans le cas de la pepsine, à savoir :

\section{TABLEAU 6}

Evolution des paramètres cinéfiques de l'hydrolyse chymotrypsique et trypsique des solutions de caséine dénaturées mécaniquement ( $3 \mathrm{~min} \mathrm{à} 50000 \mathrm{tr} / \mathrm{min}$ ) puis soumises à divers traitements thermiques pendant $20 \mathrm{~min}$

\begin{tabular}{|c|c|c|c|c|}
\hline \multirow[b]{2}{*}{$\begin{array}{c}\text { Température } \\
\text { de chauffage }\left({ }^{\circ} \mathrm{C}\right)\end{array}$} & \multicolumn{2}{|c|}{ Hydrolyse chymotrypsique } & \multicolumn{2}{|c|}{ Hydrolyse trypsique } \\
\hline & $K m_{a \mu p}(g / l)$ & $\begin{array}{c}\text { Vitesse maximum } \\
\text { d'hydrolyse } \\
\text { (g de protéine } \\
\text { hydrolysée/min) }\end{array}$ & $\mathrm{Km}_{\mathrm{app}}(\mathrm{g} / \mathrm{l})$ & $\begin{array}{l}\text { Vitesse maximum } \\
\text { d’hydrolyse } \\
\text { (g de protéine } \\
\text { hydrolysée/min) }\end{array}$ \\
\hline 37 & $6.66 \pm 0.21$ & $0,380 \pm 0,008$ & $4,61 \pm 0,20$ & $0,160 \pm 0,004$ \\
\hline 60 & $6,66 \pm 0,21$ & $0,380 \pm 0,008$ & $4,61 \pm 0,20$ & $0,160 \pm 0,004$ \\
\hline 80 & $6,41 \pm 0,21$ & $0,400 \pm 0,008$ & $4,61 \pm 0,20$ & $0,160 \pm 0,004$ \\
\hline 120 & $9,70 \pm 0,30$ & $0,250 \pm 0,007$ & $5,80 \pm 0,21$ & $0,130 \pm 0,003$ \\
\hline
\end{tabular}




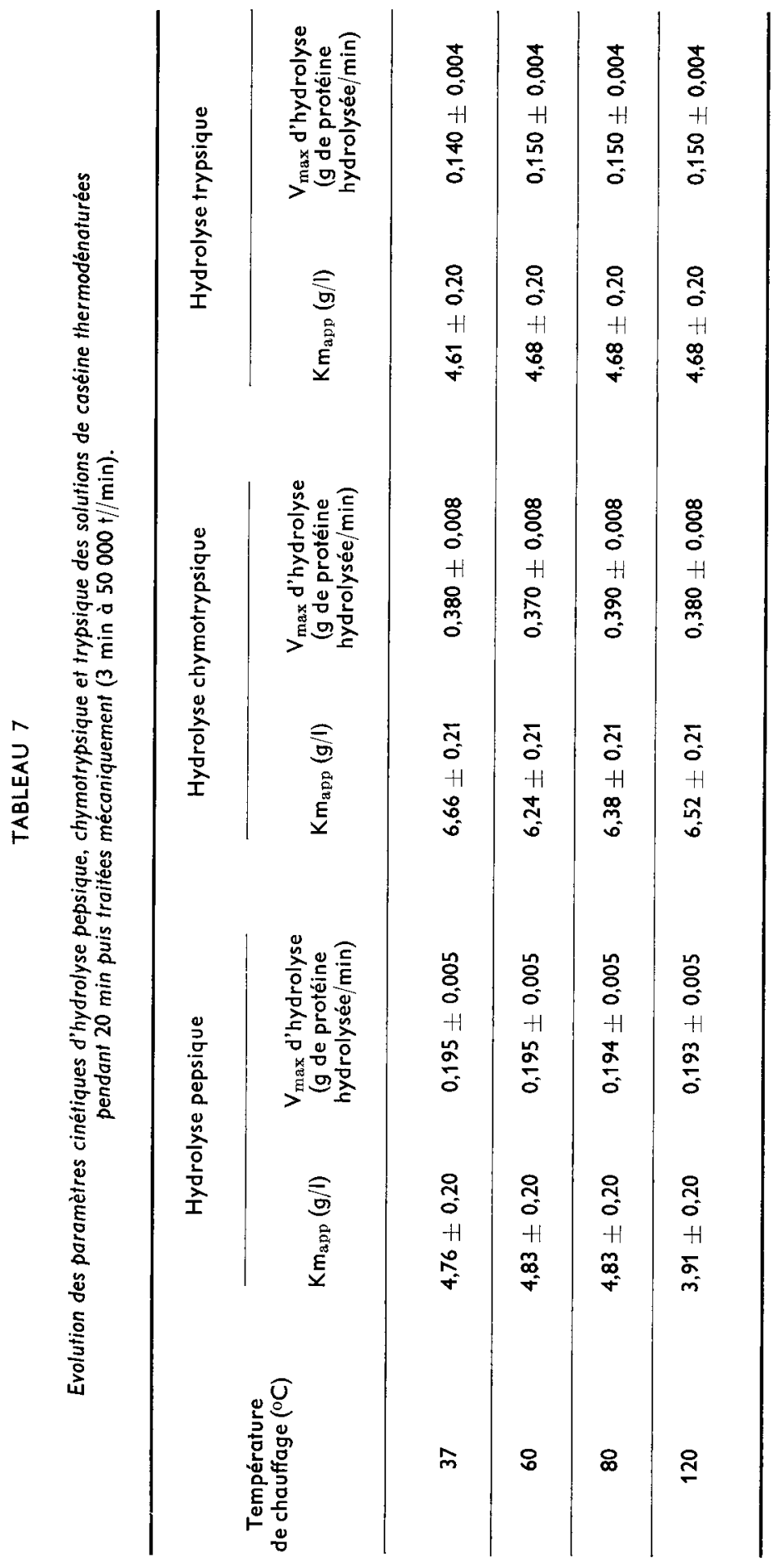


- pour des températures inférieures à $80^{\circ} \mathrm{C}$, la variation du $K m_{a p p}$ et $V_{\max }$ témoigne d'un effet de sommation des deux traitements ;

- lorsque la température atteint $120^{\circ} \mathrm{C}$, « l'effet température » classique est atténué par le traitement mécanique préalable.

\section{Incidence d'un traitement mécanique postérieur au traitement thermique}

Bien que la succession des opérations traitement thermique-traitement mécanique ne constitue pas une pratique industrielle et risque même de poser de grosses difficultés d'ordre bactériologique, il nous a paru intéressant de rechercher quelle pourrait en être l'incidence sur les protéolyses pepsiques, chymotrypsiques et trypsiques. Les résultats obtenus, rassemblés dans le tableau 7 montrent que :

- quelle que soit la température atteinte, le thermotraitement n'a plus d'effet sur les paramètres de l'hydrolyse enzymatique ;

- le traitement mécanique postérieur à une thermodénaturation à $120^{\circ} \mathrm{C}$ supprime l'effet inhibiteur que l'on constate habituellement à cette température. La comparaison des résultats des tableaux 3,6 et 7 montre que pour des températures comprises entre $37^{\circ} \mathrm{C}$ et $80^{\circ} \mathrm{C}$, la valeur des constantes cinétiques est indépendante de l'ordre des traitements.

\section{Commentaires et conclusion.}

Les traitements thermiques et mécaniques ainsi que leurs actions conjuguées influent de façon variable sur l'hydrolyse par la pepsine, la trypsine et la chymotrypsine d'une solution caséinique.

Les résultats obtenus dans nos conditions expérimentales ont permis de mettre en évidence que les vitesses de protéolyse pepsique et chymotrypsique, constantes jusqu'à $60^{\circ} \mathrm{C}$ passent par un maximum à $80^{\circ} \mathrm{C}$. II n'en est pas de même pour la protéolyse trypsique qui est maximum dès $60^{\circ} \mathrm{C}$. Lorsque la température du thermotraitement atteint $120^{\circ} \mathrm{C}$, on note au contraire pour les trois enzymes une diminution importante des vitesses d'hydrolyse. Dans ce dernier cas, et en ce qui concerne l'hydrolyse l'hydrolyse trypsique, nos résultats corroborent ceux de Swirsky ef al. (1961).

Lorsque la solution caséinique est soumise à un traitement mécanique, les vitesses maximales d'hydrolyse pepsique, trypsique et chymotrypsique augmentent respectivement de $39,2,27,2$ et 58,3 p. 100 .

Les actions conjuguées, traitement mécanique-traitement thermique ont pour effet d'amplifier de façon notable les hydrolyses pepsiques, trypsiques et chymotrypsiques par rapport aux traitements thermiques seuls.

Enfin, lorsque le traitement mécanique est postérieur au traitement thermique, les hydrolyses pepsiques, chymotrypsiques et trypsiques restent constantes et maximales, quelle que soit la température de dénaturation utilisée.

Pour expliquer un tel phénomène, plusieurs hypothèses peuvent être formulées. Un thermotraitement, à des températures ne dépassant pas $80^{\circ} \mathrm{C}$ pourrait par l'inter- 
médiaire de l'augmentation de la solubilité, ou par modification structurale du substrat faciliter l'accession des enzymes aux sites d'attaque naturels. Compte tenu des énergies nécessaires à la rupture des liaisons peptidiques ef de la relative constance des valeurs en azote peptidique TCA soluble des fractions témoins, la libération de peptides ne semble pas à priori envisageable dans nos conditions expérimentales pour expliquer l'augmentation de la protéolyse.

A $120^{\circ} \mathrm{C}$, par contre, on peut admettre avec Lorient et al. (1976) que les altérations de l'affinité enzyme-substrat constatées résultent en partie de la modification chimique des acides aminés situés au niveau des sites d'attaque enzymatiques.

Au vu des résultats, il semblerait que l'on ne puisse pas faire appel aux mêmes phénomènes pour expliquer les mécanismes d'action de la tribodénaturation. En effet, lorsque le traitement mécanique est effectué avant le thermotrailement, l'accessibilité aux sites d'attaque est augmentée comme en témoigne l'évolution des vitesses d'hydrolyse et des $\mathrm{Km}$ apparents. Lorsque le traitement mécanique est effectué après le thermotraitement, l'augmentation de la protéolyse est dans ce cas sous la seule dépendance de la tribodénaturation. Ceci sembleraił indiquer que la dénaturation consécutive au traitement mécanique entraîne essentiellement des modifications de structure moléculaire (déploiement, réarrangement) ayant pour effet une meilleure accessibilité des liaisons peptidiques. Notons aussi que même à $120^{\circ} \mathrm{C}$, les modifications d'hydrolyse observées après thermotraitement seul sont difficilement explicables par les seules altérations chimiques d'acides aminés puisque la tribodénaturation effectuée après chauffage à cette même température rétablit des constantes d'hydrolyse, voisines du maximum.

Quoi qu'il en soit, la tribodénaturation apparaît comme une technique efficace capable d'augmenter la digestibilité de solutions caséiniques. Le traitement analogue de certaines protéines végétales difficilement attaquables par les enzymes digestives peut être envisagé.

Reçu en janvier 1979.

Accepté en mai 1979.

\section{Références}

CAMUS M. C., SAUTIER C., 1972. Protéolyse pepsique d'aliments d'origine animale et végétale. Ann. Biol. anim. Bioch. Biophys., 12, 281-288.

CAUSERET J., MOCQUOT C., 1966. Effet du traitement thermique du lait sur sa valeur nutritionnelle. Cah. Nutr. Diét., 1, Fasc. 1, 31-38.

LINEWEAVER H., BURK D., 1934. Determination of enzyme dissociation constants. J. am. Chem. Soc., 56, 658.

LORIENT D., ALAIS C., 1970. Dégradation thermique des caséines $\alpha_{s}$ et $\beta$ de vache : Facteurs de variations de la dégradation. Bull. Soc. Chim. biol., 52, 915-926.

LORIENT D., ALAIS C., 1974. Dégradation thermique des caséines : Modifications des propriétés physico-chimiques. Biochimie, 56, 667-673.

LORIENT D., HUMBERT G., ALAIS C., 1976. Dégradation thermique des caséines $\alpha_{s}$ ef $\beta$ de vache : Modification de la protéolyse enzymatique : relation avec la dégradation des acides aminés. Biochimie, 58, 871-873.

ORSTEIN L., DAVIS B. J., 1964. Method and application to human serum protein. Ann. N. Y Acad. Sc., 121, 321-333 ef 404. 
OSNER R. C., 1975. Nutritional and chemical changes in heated casein. III. A comparison of the alkali-soluble and insoluble fractions. J. Fd. Technol., 10, 533-539.

OSNER R. C., JOHNSON R. M., 1975. Nutritional and chemical changes in heated casein. II. Net protein utilization, pepsin digestibility and available amino acid content. J. Fd. Technol., 10, 133-138.

ROBERT C., WARNER L., 1944. Separation of $\alpha$ and $\beta$ casein. J. am. Chem. Soc., 66, 159.

SWIRSKY M., ALLOUF R., CHEFTEL H., 1961. Contribution à l'étude de la chaleur sur les caséines. Bull. Soc. Chim. biol., 43, 909-927. 